\title{
Secondary flow in a laminar boundary layer developing over convergent-divergent riblets
}

DOI:

10.1016/j.ijheatfluidflow.2020.108598

\section{Document Version}

Accepted author manuscript

Link to publication record in Manchester Research Explorer

\section{Citation for published version (APA):}

Guo, T., Zhong, S., \& Craft, T. (2020). Secondary flow in a laminar boundary layer developing over convergentdivergent riblets. International Journal of Heat and Fluid Flow, 84, 108598.

https://doi.org/10.1016/j.ijheatfluidflow.2020.108598

\section{Published in:}

International Journal of Heat and Fluid Flow

\section{Citing this paper}

Please note that where the full-text provided on Manchester Research Explorer is the Author Accepted Manuscript or Proof version this may differ from the final Published version. If citing, it is advised that you check and use the publisher's definitive version.

\section{General rights}

Copyright and moral rights for the publications made accessible in the Research Explorer are retained by the authors and/or other copyright owners and it is a condition of accessing publications that users recognise and abide by the legal requirements associated with these rights.

\section{Takedown policy}

If you believe that this document breaches copyright please refer to the University of Manchester's Takedown Procedures [http://man.ac.uk/04Y6Bo] or contact uml.scholarlycommunications@manchester.ac.uk providing relevant details, so we can investigate your claim.

\section{OPEN ACCESS}




\title{
Secondary flow in a laminar boundary layer developing over convergent-divergent riblets
}

\author{
Tongbiao Guo*, Shan Zhong, Tim Craft \\ School of Mechanical, Aerospace and Civil Engineering, University of Manchester, Manchester M13 \\ 9PL, United Kingdom
}

\begin{abstract}
Convergent-divergent (C-D) riblets are a type of bio-inspired surface pattern. They are known to induce secondary flow in a boundary layer that may point to their potential for surface friction drag reduction and flow separation control. In this paper, a systematic investigation of the effect of riblet height, wavelength and yaw angle on the secondary flow in a flat-plate laminar boundary layer developing over a C-D riblet section is undertaken. The large-scale secondary flow in the cross-stream plane belongs to Prandtl's first kind, and is induced by the spanwise velocity generated by the yawed riblet passages. The exact structure of this secondary flow depends on the relative size of riblet height and wavelength to the local boundary layer thickness, and three different patterns are observed within the range of parameters examined. As the wavelength increases, the strength of the secondary flow increases firstly and then levels off at large wavelengths, whereas the average strength per unit area exhibits a peak around a ratio between wavelength and local boundary layer thickness of 1 . As the yaw angle increases from $20^{\circ}$ to $70^{\circ}$, the strength of the secondary flow exhibits a parabolic trend and achieves a maximum around a yaw angle of $45^{\circ}$. The findings from this work can be used to inform the choice of riblet geometry for maximizing the strength of the secondary flow in a given boundary layer flow.
\end{abstract}

Keywords: Convergent-divergent riblets; secondary flow; laminar boundary layer;

Prandtl's first kind

${ }^{*}$ corresponding author
Email address: tongbiao.guo@manchester.ac.uk. (Tongbiao Guo ) 


\section{Introduction}

Longitudinal riblets have been extensively studied since the 1980's due to their potential in reducing the surface friction drag in turbulent boundary layers from theoretical [1, 2], experimental [3, 4, 5] and numerical [6, 7, 8, 9] perspectives. They are reported to produce a reduction of skin friction drag up to $10 \%$ in flat-plate boundary layers [3, 4]. Two drag reduction mechanisms have been proposed. One mechanism is that the tips of riblets hamper spanwise fluctuations in the near-wall region leading to a reduction of skin friction drag [1, 17, 10, 11]. The other mechanism is that the near-wall streamwise vortices are trapped between the riblet tips resulting in low-speed regions inside the riblet valleys such that the shear stress over most of the ribleted surface is reduced [5, 6, 12]. Variants of longitudinal riblets have been investigated, including parallel riblets in yaw [13] and 3-D riblets [14].

Convergent-divergent (C-D) riblets are a type of nature-inspired surface roughness pattern, which are observed on the shark skins [15] and bird secondary flight feathers [16]. In recent years, C-D riblets [15, 17, 18] have begun to receive attention by researchers. As shown in the schematic diagram in Fig. 1, this type of riblet pattern is composed of sections of left-tilted and right-tilted micro-grooves arranged in an alternative manner in the span-wise direction. From the perspective of the oncoming flow, the lines along which microgrooves on adjacent riblet sections appear to diverge away from are called the diverging lines, whereas the lines on which microgrooves on adjacent riblet sections appear to converge toward each other are called the converging lines. The acute angle, which the microgrooves form with either the diverging or converging lines, is referred to as the yaw angle. It is worth noting that the yaw angle of C-D riblets on the secondary flight feathers of birds of many species is around $30^{\circ}$. Furthermore, longitudinal riblets may be regarded as the limiting case of C-D riblets with a zero yaw angle.

C-D riblets were firstly studied by Koeltzsch et al. [15] in a turbulent pipe flow, where one half of the inner surface of the pipe was covered with riblets yawed at $+45^{\circ}$ to the axial direction, and the other half was yawed at $-45^{\circ}$. From their hot-wire measurements, they found that around the converging lines the time-averaged velocity in the near-wall region decreased and the velocity fluctuations increased, whereas around the diverging lines the opposite trends occurred. In a turbulent boundary layer, Nugroho et al.19] revealed a time-averaged $\delta$-scale ( $\delta$ is the boundary layer thickness) secondary flow 
motion (also called roll mode) on cross-stream planes over each riblet strip using hot-wire measurements, as shown in the left figure of Fig. 1. Such a roll mode is manifested by a downward motion near the diverging lines that transports high-speed fluid towards the wall and an upward motion near the converging lines that takes low-speed fluid away from the wall. This consequently leads to a spanwise modulation of the boundary layer with a thinner boundary layer occurring over the diverging line and a thicker boundary layer over the converging line. The presence of such a roll mode has also been confirmed in the stereoscopic PIV experiments of Kevin et al. [18, 20] and Xu et al. [21, 22] and by Benschop et al. 23] who performed direct numerical simulations of a turbulent channel flow over C-D riblets.

Chen et al. [16] investigated the influence of C-D riblets (also called herringbone riblets) on a fully developed turbulent pipe flow. They reported a total drag reduction up to $20 \%$ which is much higher than that of traditional longitudinal riblets. Liu et al. 24] applied C-D riblets on the suction surfaces of diffuser blades and found that these riblets were capable of reducing the pressure losses in the flow passage of a linear cascade by suppressing flow separation on the blade surfaces. Quan et al. [25] also demonstrated that these riblets can be used to mitigate shock-induced flow separation in supersonic flows. These researchers attributed the control effect that they observed in their experiments to the $\delta$-scale secondary flow structures produced by the directional micro-grooves on C-D riblets.

In view of the role that the secondary flow motion produced by $\mathrm{C}-\mathrm{D}$ riblets plays in delivering the aforementioned flow control effect, it is of practical interest to understand how the strength of such flow motion could be maximized. For a given boundary layer and C-D riblets with a given riblet cross-sectional shape, the strength and appearance of this flow motion is expected to be affected by riblet geometry including riblet height, spacing, yaw angle and spanwise wavelength. Xu et al. [21] carried out a stereo PIV experiment with three different spanwise wavelengths ranging from 2.3 to 3.5 of the local undisturbed boundary layer thickness. With an increase of wavelength, they observed strengthening of the secondary flow motion and a slight outward shift of the vortex centers of this secondary flow motion on the cross-stream plane. Nugroho et al. [17] varied the relative height of riblets to the local boundary layer thickness by changing the freestream velocity in their experiments and also varied the riblet yaw angle from $10^{\circ}$ to $30^{\circ}$. They 
noticed that the secondary flow motion grows in size with an increasing riblet height or yaw angle.

In consideration of the relative narrow parameter ranges that have been studied so far and the expense of time and effort in preparing large experimental models fitted with surface patterns of a wide range of different riblet geometry patterns, a systematic investigation of the effect of riblet geometry on the characteristics of secondary flow generated by $\mathrm{C}-\mathrm{D}$ riblets using a numerical approach is hence preferable. In this study, a suitable computational method is applied to simulate the steady flow field developing along a C-D riblet section. After a grid convergence study is completed to confirm the choice of computational mesh and the computational results are validated using the experimental data from $\mathrm{Xu}$ et al. [21], the nature and development of the secondary flow motion induced by C-D riblets are examined. The effect of riblet height, wavelength and yaw angle on the secondary flow are then investigated in detail. In order to focus on studying the secondary flow motion induced by the C-D riblets without the interference from the coherent structures in a turbulent boundary layer, the simulation is undertaken in a flat-plate laminar boundary layer in this study. To the best knowledge of the authors of this paper, this is the first systematic study of the effect of riblet geometry on the characteristics of secondary flow motion generated by C-D riblets that has been undertaken so far. The findings could be used to inform the choice of riblet geometry to maximize the control effect to a given boundary layer flow.

\section{Computational Methods}

\subsection{Convergent-divergent riblets}

In Fig.1, the layout of the flat plate model is illustrated, designed to match the experimental setup of $\mathrm{Xu}$ et al. [21]. A section of $\mathrm{C}-\mathrm{D}$ riblets is inserted into the flat plate model at a certain distance from its leading edge. The smooth wall upstream and downstream of the riblets levels with the middle point of the riblets. Figure 1 also shows the coordinate system adopted in this paper, where the streamwise, wall normal and spanwise direction are denoted by $x, y$ and $z$ respectively. The origin of the coordinate system is located at the starting point of the riblet section with the $x$-axis being placed along the diverging line. The $y=0$ plane levels with the surface of the flat plate and hence the half height of the riblet. The yaw angle $(\gamma)$ is the angle between the riblet passage 
and the freestream flow direction, and the wavelength $(\Lambda)$ denotes the width of a pair of left-tilted and right-tilted riblet strips which are placed next to each other. Riblets with unilateral triangular cross-sections are used in this study and the riblet geometry is described by the height $(h)$ and spacing $(s)$, as shown in Fig. 1.

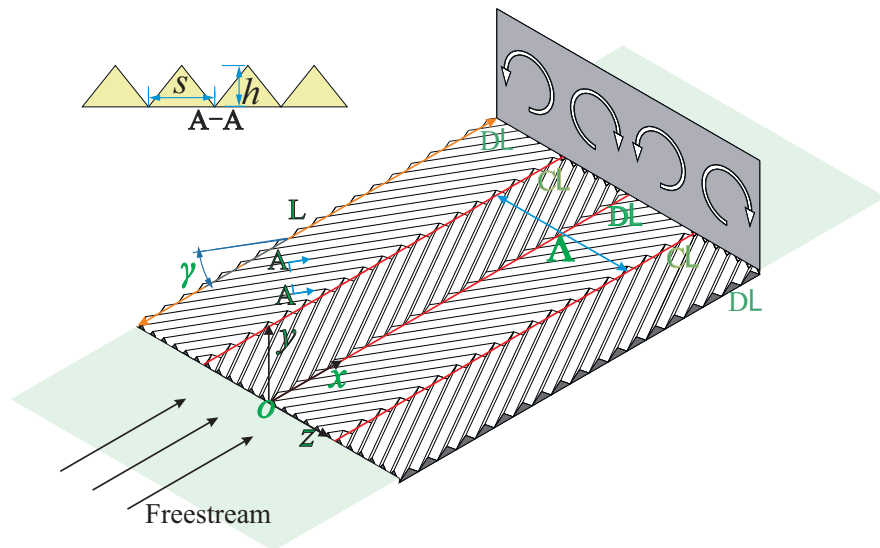

Figure 1: Schematic diagram of C-D riblets. L denotes the streamwise length of the riblet section. DL and CL are abbreviations for the diverging line and converging line respectively.

The boundary layer thickness $\delta$ is defined, based on the wall-normal coordinate $y$, as the point at which the streamwise velocity reaches $99 \%$ of its freestream value. In this paper, $\delta_{s}$ represents the local boundary layer thickness over the smooth flat plate and it is used to normalize the wall-normal coordinate $y$.

\subsection{Numerical solver, computational domain and mesh}

In the present study, the CFD software ANSYS-FLUENT is used to simulate the laminar boundary layer developing over C-D riblets at a freestream velocity of $U_{\infty}=0.1$ $\mathrm{m} / \mathrm{s}$. The pressure-based solver with constant density is adopted along with the steady laminar flow model. The numerical simulations are performed using the SIMPLE (SemiImplicit Method for Pressure-Linked Equations) algorithm, with the third-order MUSCL scheme acting as the convection discretization scheme.

The computational domain and boundary conditions are shown in Fig. 2. The inlet plane of the domain coincides with the leading edge of the flat plate and is normal to the plate. As such, the inlet velocity is assumed to be uniform and equal to the freestream velocity. The diverging line and the converging line are located at $z / \Lambda=0$ and $z / \Lambda=0.5$ respectively. A domain-independence study (not presented here for the sake of brevity) with regard to the domain height is performed for this new type of geometric pattern. 
When the domain height is larger than 10 times of the local boundary layer thickness, the flow field stays unchanged. In this paper, the domain height is set to be 15 times of the local boundary layer thickness. Due to geometric symmetry, the flow field over a riblet section with a spanwise width of only a half wavelength is simulated with symmetry boundary conditions being applied on both side planes. The working fluid is water, and a uniform velocity of $U=0.1 \mathrm{~m} / \mathrm{s}$ is applied at the inlet plane, which is located at the leading edge of the flat plate. A constant pressure condition is applied at the outlet and upper planes.

A structured mesh of hexahedral cells is adopted over the whole computational domain. In the left part of Fig. 2, the mesh topology in the wall parallel plane is displayed, where the bold light-grey lines and dark-grey lines are right above riblet tips and valleys, and the bold dark lines are right above the diverging/converging lines. The right part of Fig. 2 reveals the mesh distribution in the cross-stream plane in the vicinity of C-D riblets. It can be seen that a dense mesh is adopted near the riblet tip, while near the riblet valley, a coarse mesh is applied. A non-uniform mesh based on a geometric progression is applied in the $y$ (wall-normal) direction.

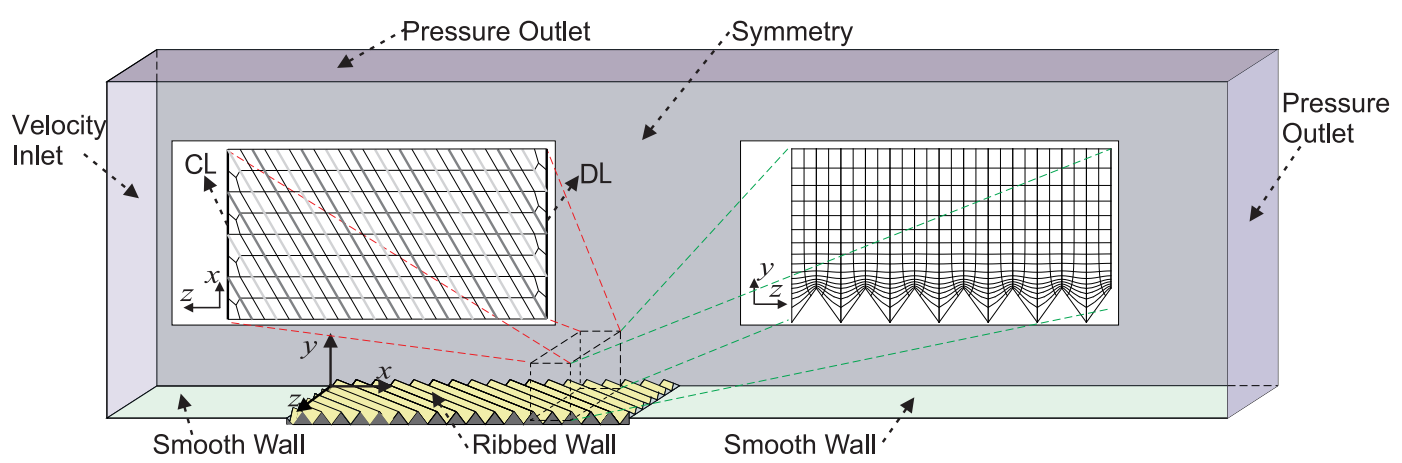

Figure 2: The computational domain and surface mesh distribution (in the wall parallel plane and the cross-stream plane).

\subsection{Validation of the computational method}

The PIV data obtained by $\mathrm{Xu}$ et al. [21] were used to validate the computational model presented here. For validation purpose, the same riblet geometry and test condition as in the experiment were adopted. The starting point of the riblet section $(x=0)$ was located $0.67 \mathrm{~m}$ downstream of the leading edge of the flat plate and the streamwise length of the C-D riblet section was $90 \mathrm{~mm}$. The local boundary layer thickness in the smooth 
wall case was $\delta_{s}=12.7 \mathrm{~mm}$ at $x=0$. The yaw angle $\gamma$ of the riblets was $30^{\circ}$, and the wavelength $\Lambda$ was $45 \mathrm{~mm}$. Triangular riblets with $s=3.0 \mathrm{~mm}$ and $h=2.4 \mathrm{~mm}$ were tested.

To ensure sufficient mesh resolution for the flow field analysis, a grid sensitive study was performed. The mesh topology in the vicinity of C-D riblets is shown in Fig. 2. For the coarse/medium/fine mesh, the mesh is plotted every 3rd/6th/9th grid line in every direction, and the corresponding total number of cells is $0.3 \mathrm{M}, 1.9 \mathrm{M}, 7.2 \mathrm{M}$ respectively. Figure 3 shows the velocity profiles at three streamwise locations over the diverging line (DL) and converging line (CL). In Fig.3(a), the velocity profiles at $x=-0.03 m$ (about $2.4 \delta_{s}$ upstream of the ribbed section) are shown and the Blasius velocity profile is also included for comparison. Based on the close agreement between the computed velocity profiles and Blasius profile, it is concluded that firstly the simulation has accurately reproduced the laminar flow field up to that location and secondly the flow field at $x=-$ $0.03 \mathrm{~m}$ is not affected by the presence of ribbed section downstream. From FIg. 3, it can be seen that the velocity profiles converge as the mesh density increases, and the profiles obtained with the 'medium mesh' and 'fine mesh' are virtually identical. Therefore, the 'medium mesh' is considered to be sufficient for resolving the velocity field. Comparing the numerical results with the experimental data from Xu et al. [21], it can be seen that the velocity profiles from the numerical simulations are in a reasonable agreement with the experimental data, verifying that the computational method applied in this paper is adequate.

Figure 4 compares the streamlines and velocity vector fields obtained from the numerical simulation and from the experiment in the cross-stream plane ( $y-z$ plane) at $x=0.075 \mathrm{~m}$. It can be seen that the simulation has reproduced well the secondary flow motion, including the presence of two weak vortex cores on this plane. Despite the challenge in accurately measuring and computing such a small magnitude of cross-stream motion, the close resemblance of the computed and measured data is reassuring, and provides a further verification of the adequacy of the computational setup used in the present study.

\subsection{Parameters of $C$-D riblets used in the parametric study}

In the experiment conducted by $\mathrm{Xu}$ et al. [21], relatively large riblets were used to facilitate their dye flow visualization experiment, and the streamwise length of the riblet section $(0.09 \mathrm{~m})$ was quite short due to experimental constraints. To obtain a more 


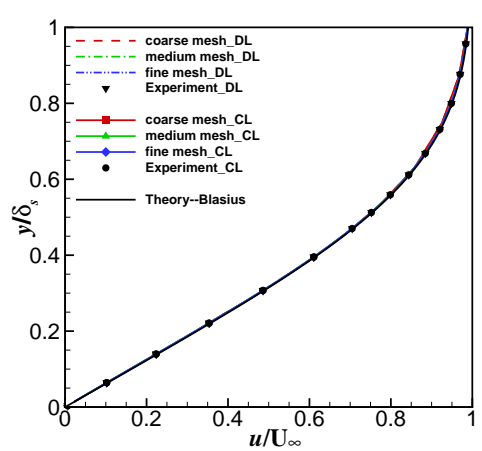

(a) $x=-0.03 m$

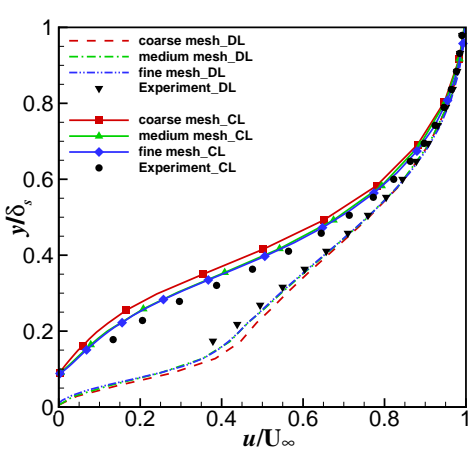

(b) $x=0.045 \mathrm{~m}$

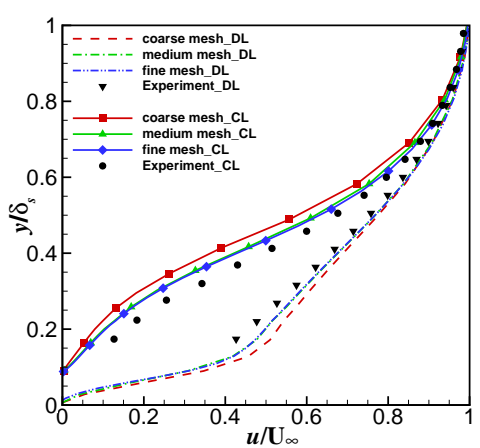

(c) $x=0.075 \mathrm{~m}$

Figure 3: Comparison of the streamwise velocity profiles obtained with different mesh densities against experimental data and Blasius profile over the diverging line (DL) and the converging line (CL). The experimental results are extracted from [21].

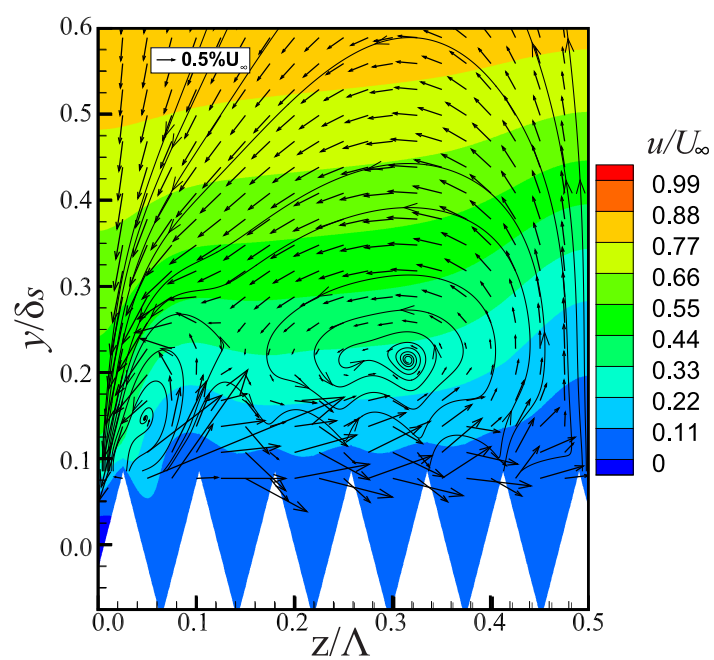

(a) Present simulation

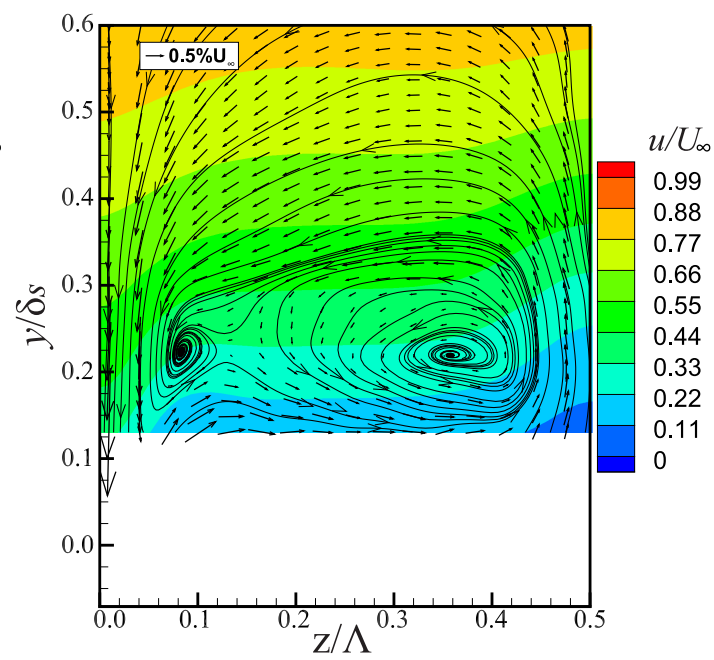

(b) Experimental results in 21 .

Figure 4: Streamlines and velocity vectors in the cross-stream plane at $x=0.075 \mathrm{~m}$. 'DL' is located at $z / \Lambda=0$ and 'CL' at $z / \Lambda=0.5$.

fully developed roll mode, closer to what might be seen in real applications, a longer length riblet section of $L=0.27 \mathrm{~m}$ is used in the present simulations. To offset some of the computational cost associated with the use of a longer riblet section, the upstream section of the flat plate is shortened to $0.4 \mathrm{~m}$, which is still sufficient to give a developed laminar boundary layer of thickness $9.84 \mathrm{~mm}$ just before the start of the riblet section at a freestream velocity of $0.1 \mathrm{~m} / \mathrm{s}$.

In the parameter study presented in this paper, the effects of riblet height, wavelength and yaw angle on the secondary flow are investigated. Table 1 presents the wavelengths examined for a fixed riblet height, spacing and yaw angle. Table 2 reveals the riblet 
heights examined for a fixed riblet spacing and yaw angle, for two different values of riblet wavelength. Table 3 lists the yaw angles examined for a fixed riblet height, spacing and wavelength. The baseline (referred to as Case 0 in the discussion below) is the case of a flat surface, with no riblets.

With the computational method having been validated, a similar computational approach and mesh setting strategy are used to compute the flow field in cases with different riblet geometries. Additional grid-sensitivity studies are carried out to ensure that the grid resolution is still sufficient, although for the sake of brevity those results are not presented here.

Table 1: Parameters of C-D riblets with different wavelengths $\left(\gamma=30^{\circ}, s=0.75 \mathrm{~mm}\right.$ and $\left.h=0.6 \mathrm{~mm}\right)$.

\begin{tabular}{|c|c|c|c|c|c|c|c|c|c|c|}
\hline Case No. & Case 1 & Case 2 & Case 3 & Case 4 & Case 5 & Case 6 & Case 7 & Case 8 & Case 9 & Case 10 \\
\hline$\Lambda(\mathrm{mm})$ & 1.7 & 5.2 & 8.7 & 12.1 & 15.6 & 31.2 & 46.8 & 62.4 & 78.0 & 93.6 \\
\hline
\end{tabular}

Table 2: Parameters of C-D riblets with different riblet heights $\left(\gamma=30^{\circ}, s=0.75 \mathrm{~mm}\right)$.

\begin{tabular}{|c|c|c|c|c|}
\hline & \multicolumn{2}{|c|}{$\Lambda=15.6 \mathrm{~mm}$} & \multicolumn{2}{c|}{$\Lambda=46.8 \mathrm{~mm}$} \\
\hline Case No. & Case 5 & Case 5b & Case 7 & Case 7b \\
\hline$h(\mathrm{~mm})$ & 0.6 & 2.4 & 0.6 & 2.4 \\
\hline
\end{tabular}

Table 3: Parameters of C-D riblets with different yaw angles $(\Lambda=15.6 \mathrm{~mm}, \mathrm{~s}=0.75 \mathrm{~mm}$ and $h=0.6 \mathrm{~mm})$.

\begin{tabular}{|c|c|c|c|c|c|c|c|}
\hline Case No. & Case 5A & Case 5 & Case 5C & Case 5D & Case 5E & Case 5F & Case 5G \\
\hline$\gamma$ & $20^{\circ}$ & $30^{\circ}$ & $40^{\circ}$ & $45^{\circ}$ & $50^{\circ}$ & $60^{\circ}$ & $70^{\circ}$ \\
\hline
\end{tabular}

\section{Results and discussion}

\subsection{General influence of riblets on a laminar boundary layer}

Before considering the detailed parametric study, this section examines the general qualitative effects of C-D riblets on the development of a laminar boundary layer, including the fluid motion inside the riblet valley and the flow field in a cross-stream plane $(y-z$ plane). Case $5\left(h=0.6 \mathrm{~mm}, \Lambda=15.6 \mathrm{~mm}, \gamma=30^{\circ}\right)$ is selected for this study. 


\subsubsection{Fluid motion inside riblet valleys}

Figure 5 shows the spatial streamlines of the flow inside riblet valleys and the in-plane streamlines in a plane perpendicular to the riblet passage. From the spatial streamlines it can be seen that firstly some fluid near the surface is fed into the riblet passage from above the diverging line and it travels along the riblet passage before emerging from above the converging line. Secondly, the fluid inside the riblet passage travels along the riblet passage in a helicoidal manner (clockwise rotation). Flow recirculating motion revealed by the streamline pattern in the plane perpendicular to the riblet passage also confirms the existence of the helicoidal motion. Such a finding is consistent with the observation of $\mathrm{Xu}$ et al. [21] in their dye visualization experiment.

It is clear that firstly the specific directional orientation of the riblet passages plays a key role in generating such a surface flow motion from the diverging line to converging line. Secondly, the high-momentum flow above the riblet passages helps sustaining the helicoidal motion inside the riblet passage through its cross-flow motion. Such a cross-flow motion can be decomposed into two velocity components: one along the riblet passage which contributes to the axial component of the helicoidal motion, and the other perpendicular to the riblet passage which contributes to the rotational component of the helicoidal motion.

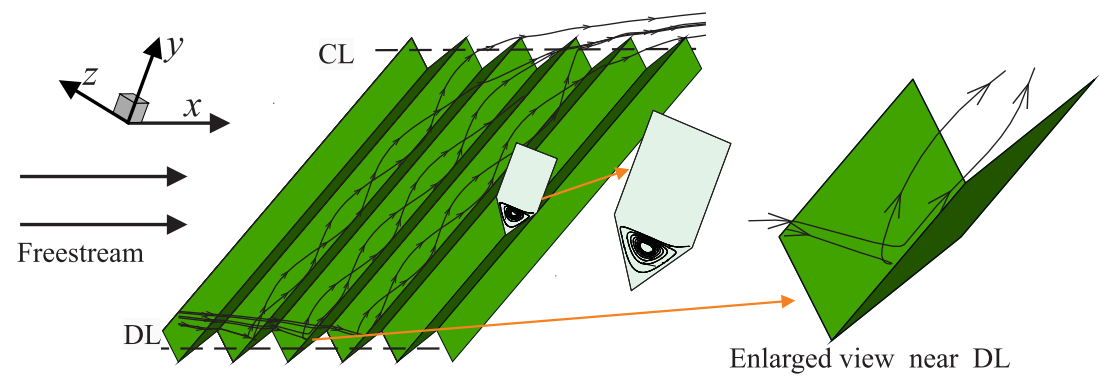

Figure 5: Streamlines inside the riblet valleys and in the plane perpendicular to the riblet passage. 'CL' denotes the converging line, whereas 'DL' denotes the diverging line.

\subsubsection{Flow characteristics in the cross-stream plane}

Figure 6(a) presents velocity vectors and streamlines along with contours of streamwise vorticity in the cross-stream plane $(y-z$ plane $)$ at $x=0.18 \mathrm{~m}$. It shows that a largescale roll motion is developed which displays a downwash motion over the diverging line, an upwash motion over the converging line and a span-wise motion directing from the diverging line to the converging line over the riblets. With the spanwise flow generated 
by the yawed riblets from the diverging line to the converging line near the wall, to satisfy the mass conservation, a downward motion and an upward motion hence occur near the diverging and converging line respectively and a secondary recirculating motion cross the boundary layer is produced. Due to geometric symmetry, there exists a pair of counterrotating recirculating motions over a full riblet wavelength. Such a large-scale roll mode has also been observed both in laminar flow [21] and turbulent flow [18].

Prandtl classified secondary flows into two categories based on their generation mechanisms [26]. Prandtl's first kind, also known as geometry-driven secondary flow, is induced due to the misalignment between the direction of wall-mounted geometric pattern and the crossflow, and it can observed in both laminar flows and turbulent flows. By contrast, Prandtl's second kind, also known as turbulence-driven secondary flow, is induced by the heterogeneity of turbulence [27], which can only be obtained in turbulent flows. For the laminar boundary layer flow considered in this study, the large-scale roll mode observed in the cross-stream plane is induced by the yawed riblets, and hence belongs to the secondary flow of Prandtl's first kind.

In order to examine the vertical velocity induced by the riblets, contours of the difference in the wall-normal velocity in the ribbed case and the baseline case are shown in Fig. 6(b). The maximum induced downwash is observed over the diverging line and is about $0.4 \% U_{\infty}$, whereas the induced upwash over the converging line is about $0.2 \% U_{\infty}$. The influence of C-D riblets on the wall-normal velocity is largely confined near the diverging/converging lines. Noticeable local alternation of the vertical velocity is also observed near the riblet tips as the surface flow is riding cross the riblets. In the bulk region away from the diverging line and the converging line, the induced wall-normal velocity is almost zero.

It is worth noting that although the large-scale roll motion in the cross-stream plane is clear from Fig. 6(a), its strength is quite weak, with a velocity magnitude away from the immediate riblet vicinity being less than $0.3 \% U_{\infty}$ in this case. Furthermore, there is considerable offset between the region of high vorticity and the recirculating motion revealed by the streamlines. In the case of a conventional vortex generator, the region of high vorticity produced by the vortex generator normally coincides with the region of high swirling motion. Hence the vortical flow generated by C-D riblets exhibits a different nature to that of the vortex generated by a conventional vortex generator; a 
distinction that should not be overlooked. Nevertheless, as will be shown later, even this weak secondary flow does produce a profound modification in the boundary layer.

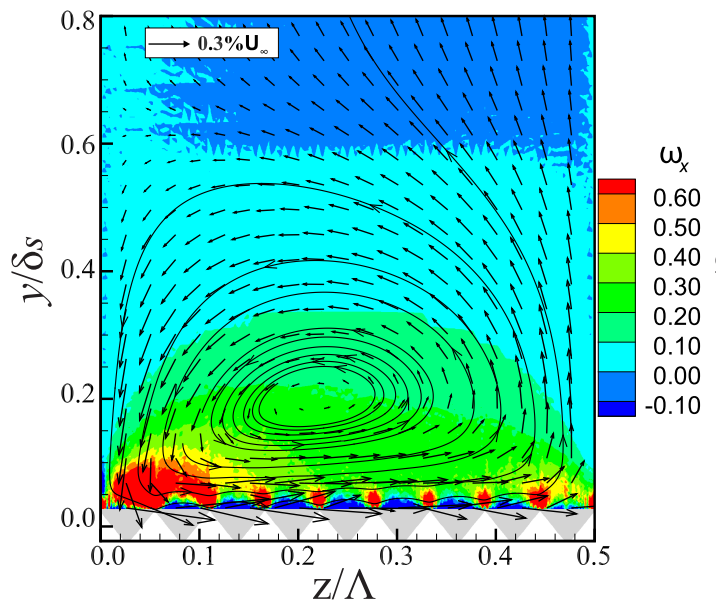

(a)

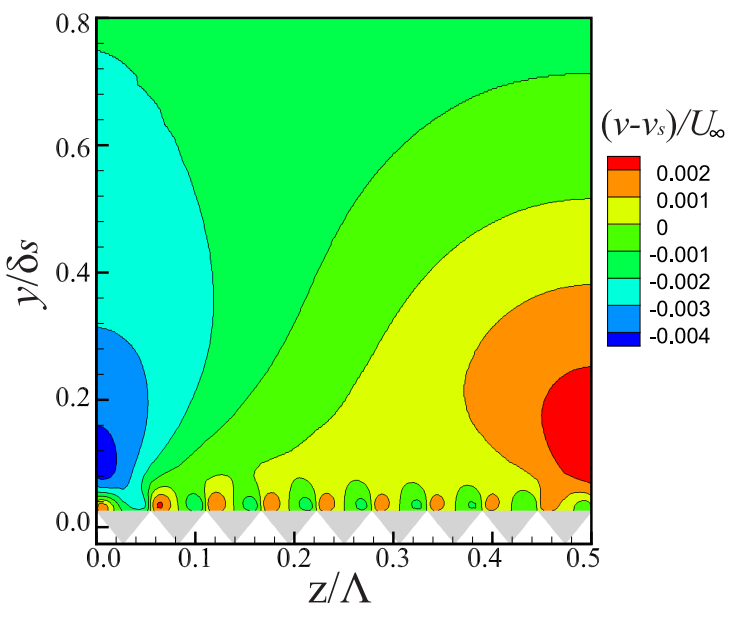

(b)

Figure 6: Streamwise vorticity and in-plane velocity field in the cross-stream plane $x=0.18 \mathrm{~m}$ for Case 5. (a) Contours of streamwise vorticity and in-plane velocity vectors. (b) Contours of induced wallnormal velocity. $v$ is the wall-normal velocity for riblet cases, and $v_{s}$ is the wall-normal velocity at the corresponding location for the flat plate, Case 0 . 'DL' is located at $z / \Lambda=0$, while 'CL' is located at $z / \Lambda=0.5 . \quad \delta_{s}=11.8 \mathrm{~mm}$ is the local boundary layer thickness of Case 0 at $x=0.18 \mathrm{~m}$.

\subsubsection{Development of roll mode}

To examine the streamwise development of the roll mode, contours of the streamwise velocity and in-plane velocity vectors on a number of cross-stream planes are shown in Fig. 7. It is observed that as the boundary layer over the ribbed surface develops in the streamwise direction, both the intensity and the extent of the roll motion increase. In addition, the center of the circulating motion gradually moves away from the wall surface and towards the middle point between the converging and diverging lines. For example, at $x=0.015 \mathrm{~m}$ (Fig. $7(\mathrm{a}))$ the roll mode is confined near the ribbed wall within $y / \delta_{s}<0.3$ and the center of the roll mode lies at approximately $y / \delta_{s}=0.1$, whilst the bulk region further away from the wall appears unaffected. In contrast, at $x=0.12 m$ (Fig. $7(\mathrm{~d})$ ) the roll motion is extended across more than $50 \%$ of the boundary layer thickness, and an obvious spanwise variation in the streamwise velocity is observed nearly to the edge of the boundary layer.

Although the qualitative development of the roll mode in the streamwise direction can be clearly observed in Fig. 7, it is worth quantifying its intensity using an adequate 


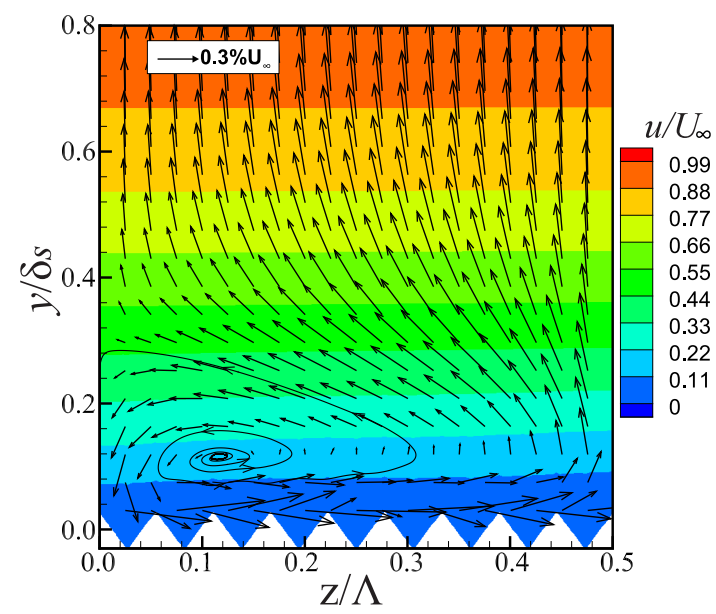

(a) $x=0.015 m$

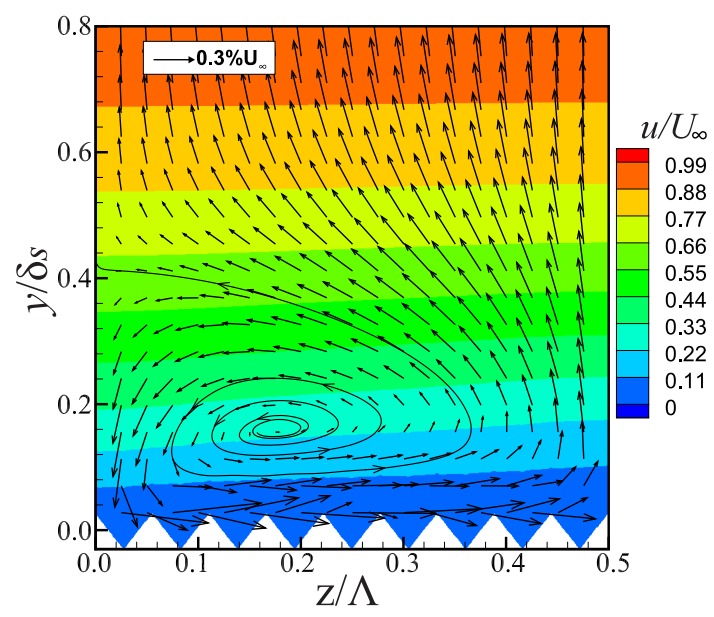

(c) $x=0.060 m$

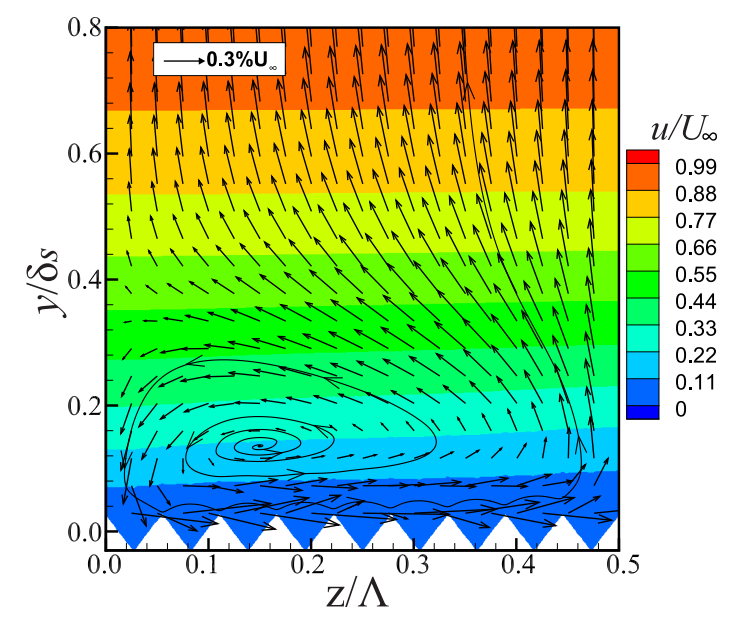

(b) $x=0.030 m$

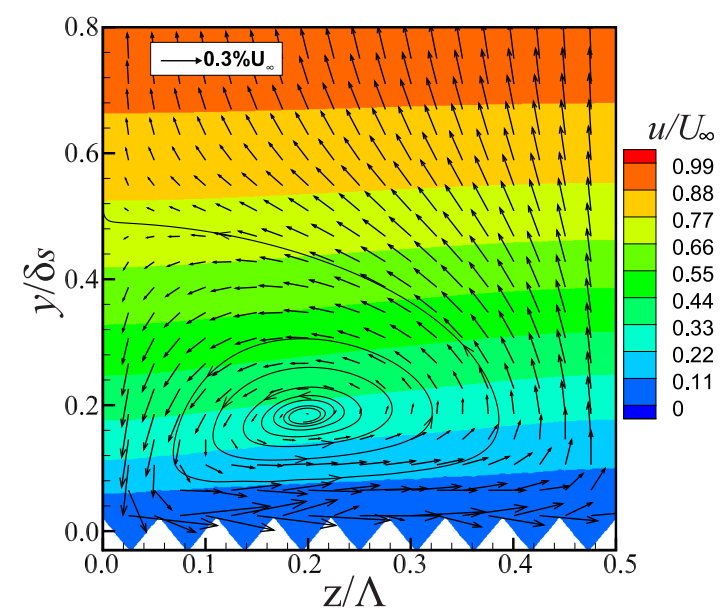

(d) $x=0.120 m$

Figure 7: Contours of the streamwise velocity and in-plane velocity vectors on cross-stream planes at different streamwise locations in Case $5 . \quad \delta_{s}$ is the local boundary layer thickness of Case 0 for each streamwise position.

parameter to enable a better comparison between different cases. Swirling strength, $\lambda_{c i}$, is the imaginary part of the complex eigenvalue of the velocity gradient tensor, and it can be used to indicate the strength of local vortical motion [28]. In order to indicate the direction of rotation of swirl, signed swirling strength [29] $\Lambda_{c i}$ is obtained by multiplying $\lambda_{c i}$ by the sign of the streamwise vorticity $\omega_{x} /\left|\omega_{x}\right|$, i.e. $\Lambda_{c i}=\lambda_{c i} \omega_{x} /\left|\omega_{x}\right|$. Figure $8($ a) shows contours of the signed swirling strength in the cross-stream plane at $x=0.18 m$, where the swirling strength $\lambda_{c i}$ is the imaginary part of the complex eigenvalue of the twodimensional $\left(y\right.$ and $z$ ) velocity gradient tensor. $\Lambda_{c i}>0$ corresponds to an anticlockwise rotation, whereas $\Lambda_{c i}<0$ represents a clockwise vortical motion. From Fig. 8(a), it can be seen that the roll mode approximately coincides with a zone of relatively high positive 
value of $\Lambda_{c i}$ in the cross-stream plane.

The total strength of the secondary flow motion created by the riblets at each streamwise location can then be quantified by integrating $\lambda_{c i}$ over the cross-stream plane:

$$
\hat{\lambda}_{c i}=\int_{\text {riblet tip }}^{y=\delta_{s}} \int_{z=0}^{z=0.5 \Lambda} \lambda_{c i} d y d z
$$

Figure $8(\mathrm{~b})$ shows the streamwise variation of the total swirling strength $\hat{\lambda}_{c i}$. It can be seen that $\hat{\lambda}_{c i}$ experiences a rapid increase at the beginning of the riblet section and a rapid decrease towards the end of the riblet section. Such a rapid change in $\hat{\lambda}_{c i}$ takes place over a streamwise distance equivalent to about five times of the local boundary layer thickness. In the middle of the riblet section, $\hat{\lambda}_{c i}$ is almost constant. Towards the end of the riblet section, the ability of C-D riblets in generating a spanwise flow directing from the diverging line to the converging line is compromised due to the shortening of the grooves. As a result, the strength of secondary flow decreases accordingly. In the following analysis, the results will be presented at $x=0.18 m$ where $\hat{\lambda}_{c i}$ changes only very slowly.

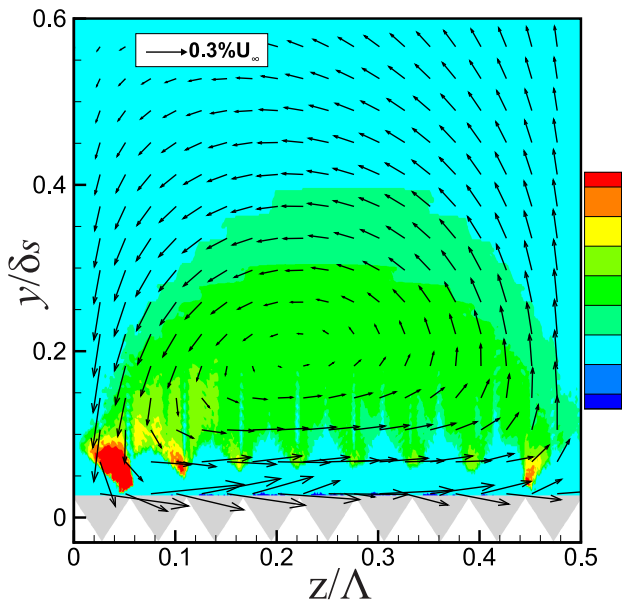

(a) The distribution of $\Lambda_{c i}$ at $x=0.18 m$

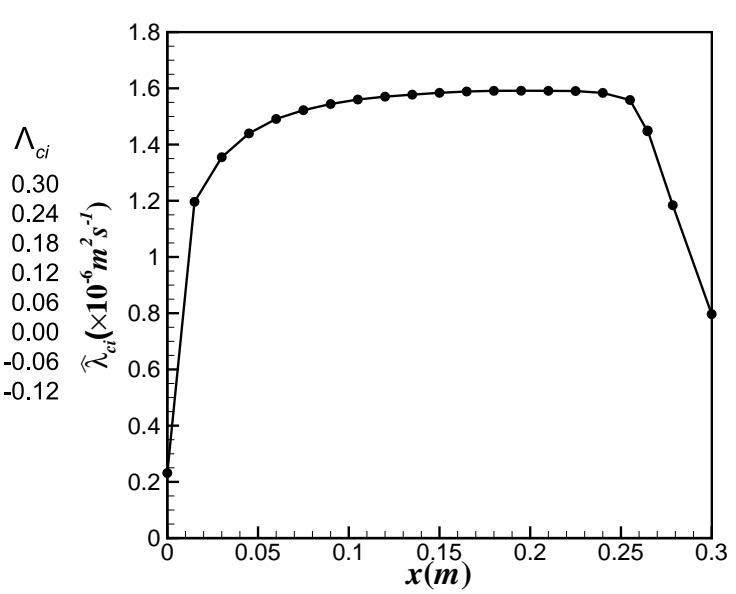

(b) $\hat{\lambda}_{c i}$ along the streamwise direction

Figure 8: Swirling strength in the cross-stream planes for Case 5. $\Lambda_{c i}$ is the signed swirling strength. $\hat{\lambda}_{c i}$ is the total swirling strength across the corresponding cross-stream plane. $\delta_{s}$ is the local boundary layer thickness of Case 0 at $x=0.18 \mathrm{~m}$.

\subsection{The effect of riblet height}

In this section, the effect of the riblet height on the roll mode and the boundary layer is analysed for two different wavelengths, i.e. $\Lambda=15.6 \mathrm{~mm}\left(\Lambda / \delta_{s}=1.32\right)$ and $\Lambda=46.8 \mathrm{~mm}$ 
$\left(\Lambda / \delta_{s}=3.97\right)$ (see Table 2$)$. Here and hereafter, the results presented are from the crossstream plane at $x=0.18 \mathrm{~m}$, where the local boundary layer thickness of the baseline case is $\delta_{s}=11.8 \mathrm{~mm}$. Two riblet heights, $5 \%$ and $20 \%$ of $\delta_{s}$, are examined, while the spacing between the riblet tips remains unchanged.

The effect of the riblet height on the contours of the streamwise velocity and in-plane velocity vector in the $y-z$ plane is illustrated in Fig. 9. For a given riblet wavelength, with an increase in riblet height, the magnitude of in-plane velocity and the amount of spanwise variation in the streamwise velocity tend to increase, indicating an increase in the intensity of secondary flows induced by C-D riblets.

For Case 7 with $\Lambda / \delta_{s}=3.97$ and $h=0.6 \mathrm{~mm}$, a large-scale roll mode centred around $z / \Lambda=0.2$ is observed (see Fig. $9(\mathrm{a})$ ), which is caused by the global spanwise flow motion directing from the diverging line to the converging line generated by the yawed riblets. As the height of the riblets is increased to $h=2.4 \mathrm{~mm}$ (Case $7 \mathrm{~b}$ ), a pair of co-rotating vortical structures is observed (see Fig. 9(b)), which is consistent with the finding by Xu et al. [21] who studied riblets with a similar $\Lambda / \delta_{s}$ and riblet height in their experiment. Compared with Case 7 (Fig. 9(a)), the downwash near the diverging line in Case 7b is much stronger due to a larger riblet height (Fig. 9(b)). When the strong downwash strikes the upstream facing side of the riblet valley, the fluid is deflected upwards and away from the diverging line (see Fig. 5). This local motion is manifested by the inclined upward pointing in-plane velocity vectors near the diverging line over the riblet surface in Fig. 9(b). Under the combined influence of this local upward motion and the strong downwash over the diverging line, a small local vortex is developed over the riblet surface and very close to the diverging line. The larger vortex on the right is caused by the global spanwise flow motion directing from the diverging line to the converging line, similar with that observed in Case 7 (see Fig. 9(a)).

As the wavelength is reduced to $\Lambda / \delta_{s}=1.32$, a similar large-scale roll mode is still observed in Case 5 (Fig. 9(c)) as in Case 7 when the riblet height is small $(h=0.6 \mathrm{~mm})$, although the vortex center in Case 7 appears to locate nearer to the diverging region. When the riblet height is increased to $h=2.4 \mathrm{~mm}$, in contrast to Case 7b only one vortex is observed in Case 5b (see Fig. 9(d)). It is likely that the strong local motion near the diverging line observed in Case $5 \mathrm{~b}$ has amalgamated with the global motion to form a single vortex as a result of a significant reduction in the wavelength. 

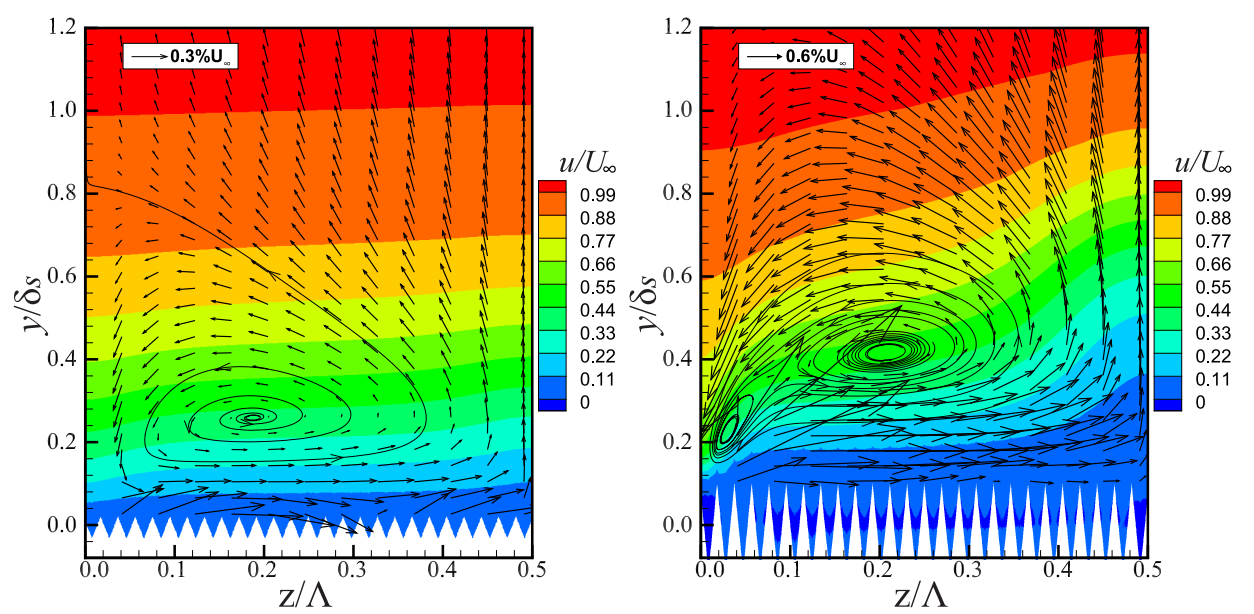

(a) Case 7: $\Lambda / \delta_{s}=3.97, h=0.6 \mathrm{~mm}$

(b) Case 7b: $\Lambda / \delta_{s}=3.97, h=2.4 \mathrm{~mm}$
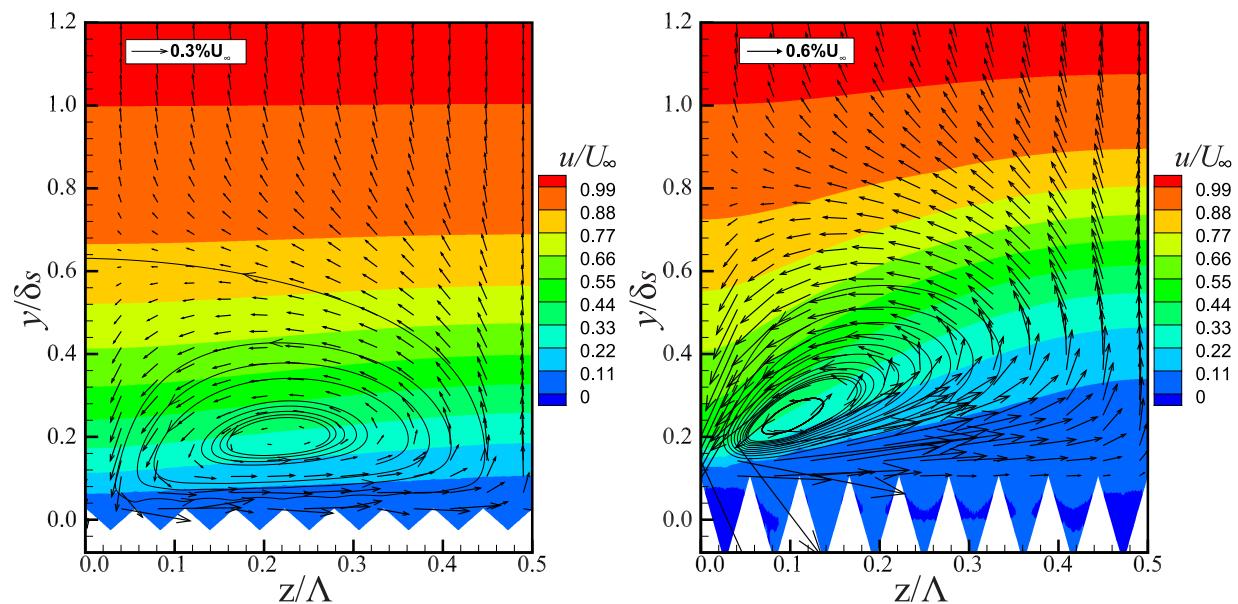

(c) Case 5: $\Lambda / \delta_{s}=1.32, h=0.6 \mathrm{~mm}$

(d) Case 5b: $\Lambda / \delta_{s}=1.32, h=2.4 \mathrm{~mm}$

Figure 9: Comparison of contours of the streamwise velocity and velocity vectors in the cross-section at $x=0.18 m$ for cases with different heights. $\delta_{s}$ is the local boundary layer thickness of Case 0 at $x=0.18 \mathrm{~m}$.

\subsection{The effect of riblet wavelength}

In this section, the effect of riblet wavelength on the laminar boundary layer is studied with $\Lambda / \delta_{s}$ changing from 0.14 to 7.93 . Triangular riblets with $\gamma=30^{\circ}, s=0.75 \mathrm{~mm}$ and $h=0.6 \mathrm{~mm}$ are used. The geometric parameters for each case are shown in Table 1.

Figure 10 presents contours of streamwise velocity and in-plane velocity vectors in the cross-stream plane at $x=0.18 \mathrm{~m}$. In each case, a large-scale streamwise vortex can be clearly observed over a half wavelength.

Due to geometric symmetry, the spanwise velocity in the longitudinal planes $(x-y$ planes) over the diverging line and the converging line is constrained to be zero. According to the above, it is the spanwise motion induced by yawed ribles in the near wall region that drives the large-scale secondary motion. For cases with a small wavelength, since 


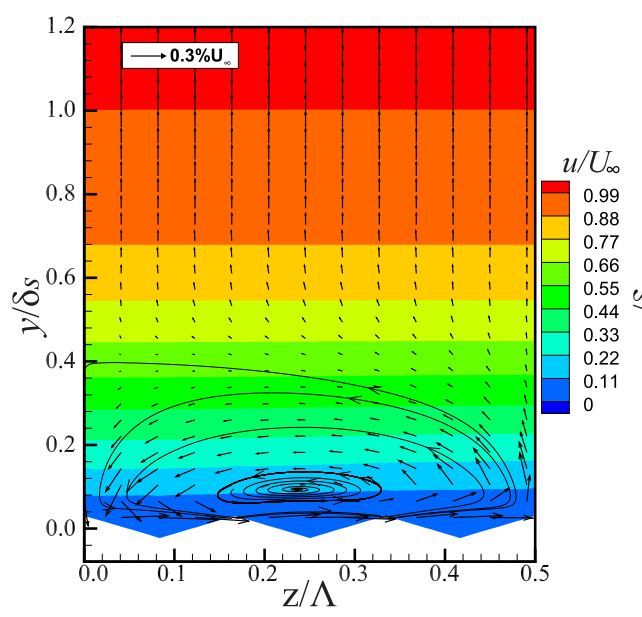

(a) Case 2: $\Lambda / \delta_{s}=0.44$

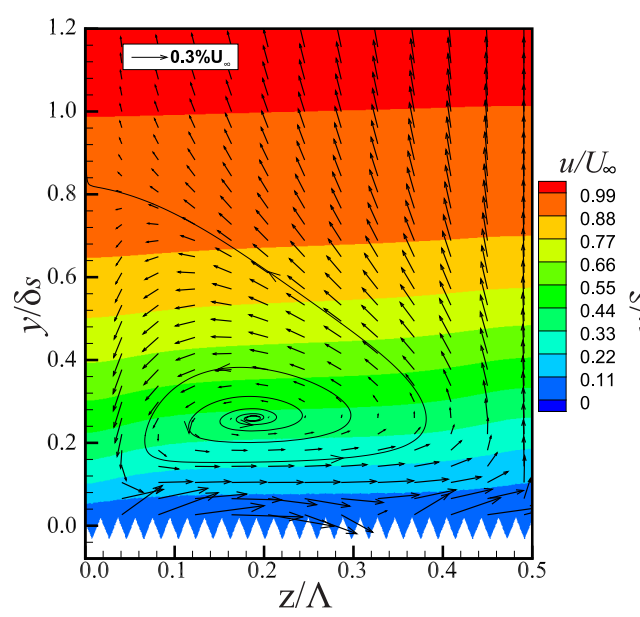

(c) Case 7: $\Lambda / \delta_{s}=3.97$

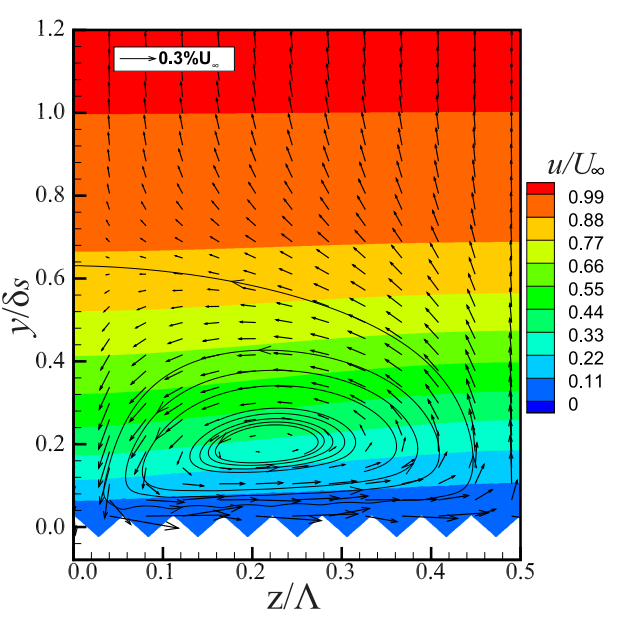

(b) Case 5: $\Lambda / \delta_{s}=1.32$

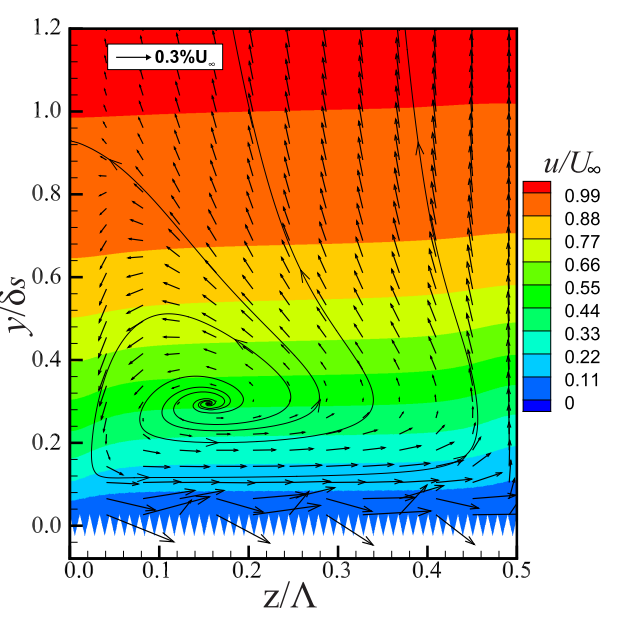

(d) Case 9: $\Lambda / \delta_{s}=6.61$

Figure 10: Comparison of contours of the streamwise velocity and in-plane velocity vector in the crossstream plane at $x=0.18 \mathrm{~m}$ for riblets of different wavelengths. For all cases, $\gamma=30^{\circ}, s=0.75 \mathrm{~mm}$ and $h=0.6 \mathrm{~mm} . \delta_{s}$ is the local boundary layer thickness of Case 0 at $x=0.18 \mathrm{~m}$.

there is a limited spanwise length to enable the spanwise velocity to develop between these two planes, the constraint of the zero-spanwise velocity could have an impact across the whole wavelength. Consequently, the spanwise velocity and the strength of the secondary flow tend to be quite weak. This seems to be the case for Case 2 with $\Lambda / \delta_{s}=0.44$. The large-scale roll mode is confined in the near-wall region within $y / \delta_{s}<0.4$ and the vortex center lies below $y / \delta_{s}=0.1$ (Fig. 10(a)). Although the downward/upward motion occurs near the diverging/converging region, the strength of the secondary flow is too weak to result in a noticeable spanwise variation of the streamwise velocity.

With an increase in wavelength, the constraint on the establishment of spanwise velocity is gradually reduced, and thus the spanwise motion and the strength of the secondary 
flow tend to increase. The size of roll motion also increases with the vortex center gradually moving away from the wall surface. For Case 5 with $\Lambda / \delta_{s}=1.32$, the large-scale roll mode extends itself across more than $60 \%$ of the whole boundary layer with its center lying at $y / \delta_{s} \approx 0.2$ (Fig. $10(\mathrm{~b})$ ). In addition, an obvious spanwise variation of the streamwise velocity is observed with increased/decreased streamwise velocity appearing around the diverging/converging region. For cases with a large wavelength, Case 9 for example, the vortex extends itself nearly across the whole boundary layer (Fig. 10(d)). This leads to the spanwise variation not only in the streamwise velocity but also in the boundary layer thickness, which is not observed in Case 5. Besides, for Case 9, the spanwise variation of the streamwise velocity mainly occurs near the diverging/converging region, whereas in the bulk region away from the diverging and converging line, little spanwise variation is present. This indicates that for cases with large wavelength, the downward/upward motion mainly occurs and has an effect around the diverging/converging region.

In Fig. 11 the profiles of streamwise velocity at $x=0.18 \mathrm{~m}$ over the diverging line and converging line are compared. As the wavelength increases, the streamwise velocity profile over the diverging line becomes fuller and the opposite occurs for the velocity profiles over the converging line. This results from the increase of the induced downward/upward motion near the diverging/converging region. It is also worth noting that the streamwise velocity profiles tend to become indistinguishable as the wavelength increases above $\Lambda / \delta_{s} \geq 3.97$. This indicates that for cases with large wavelength $\left(\Lambda / \delta_{s} \geq 3.97\right)$, the streamwise velocity profiles over the diverging/converging line tend to become independent with the wavelength, and thus the strength of the downward/upward motion near the diverging/converging line that induces the modification of the streamwise veloctity is also expected to become independent with the wavelength.

Figure 10 presents qualitative comparison of the strength of the secondary flow among changed wavelengths. To quantitatively compare the strength of the secondary flow for these cases, the total swirling strength $\hat{\lambda}_{c i}$ defined in Eq. (1) is computed and shown in Fig. 12. It can be seen in Fig. 12(a) that $\hat{\lambda}_{c i}$ increases monotonically with an increasing wavelength with a fast rate occurring at small wavelengths. This is because that the constraint on the establishment of spanwise velocity that drives the large-scale roll mode is gradually reduced as the wavelength increases. At large wavelengths, the constraint of the zero-spanwise velocity in the $x-y$ planes over the diverging line and the converging 


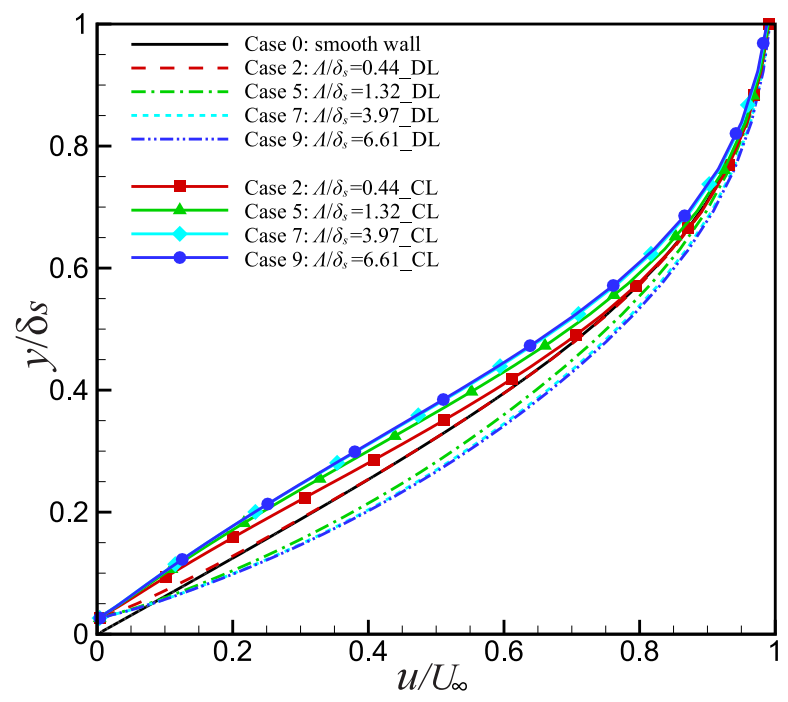

Figure 11: Profile of the streamwise velocity over the diverging line (DL) and over the converging line (CL) at $x=0.18 m$ for riblets of different wavelengths. $\delta_{s}$ is the local boundary layer thickness of Case 0 at $x=0.18 \mathrm{~m}$. For all cases, $\gamma=30^{\circ}, s=0.75 \mathrm{~mm}$ and $h=0.6 \mathrm{~mm}$.

line is applied near the two longitudinal planes, with the bulk region between the two planes being free from the constraint. Consequently, the influence of wavelength on the strength of the secondary flow will gradually disappear as the wavelength further increases. Therefore, the strength of roll mode tends to level off and become independent of the wavelength.

Since the total swirling strength increases with the size of integration area, another parameter, $\bar{\lambda}_{c i}$, which indicates the average swirling strength per unit area, is introduced,

$$
\bar{\lambda}_{c i}=\frac{\hat{\lambda}_{c i}}{0.5 \Lambda \delta_{s}}
$$

As shown in Fig. 12(b), $\bar{\lambda}_{c i}$ increases with wavelength until about $\Lambda / \delta_{s}=1.03$ and it then starts to decrease. It is worth mentioning that Chung et al. [30] performed direct numerical simulations of a turbulent channel flow bounded by walls fitted with spanwise alternating patches of high and low wall shear stress. In their study, the roll motion in the cross-stream plane is caused by the heterogeneity of turbulence, and hence belongs to the secondary flow of Prandtl's second kind. They found that the roll motion is most pronounced when the spanwise wavelength equals approximately to the boundary layer thickness. For the present study, the large-scale roll mode belongs to the secondary flow of Prandtl's first kind, and a similar result is obtained. Therefore, the wavelength is key parameter to maximum the strength of the secondary flow for the secondary flow of both 
Prandtl's first kind and second kind. This finding could be useful in guiding the choice of spanwise wavelength for practical applications.

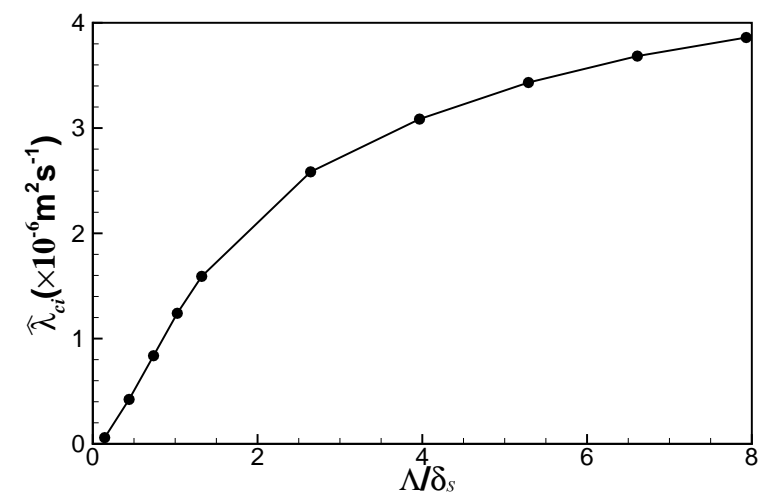

(a) Integrated swirling strength

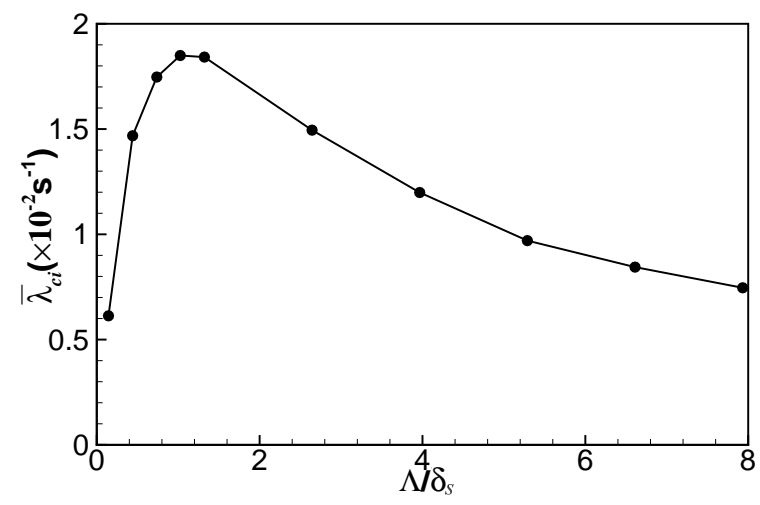

(b) Average swirling strength

Figure 12: Integrated and average swirling strength in the cross section $x=0.18 \mathrm{~m}$ for cases with different wavelengths. For all cases, $\gamma=30^{\circ}, s=0.75 \mathrm{~mm}$ and $h=0.6 \mathrm{~mm}$.

\subsection{The effect of yaw angle}

In this section, the effect of yaw angle on the boundary layer is analyzed with the yaw angle varying from $20^{\circ}$ to $70^{\circ}$. Triangular riblets with $\Lambda / \delta_{s}=1.32, s=0.75 \mathrm{~mm}$ and $h=0.6 \mathrm{~mm}$ are used. The geometry parameters of each case are shown in Table 3.

Figure 13 presents contours of streamwise velocity and velocity vectors in the crossstream plane at $x=0.18 m$ for these cases. In each case, a large-scale roll motion can be clearly observed over a half wavelength. Both the strength of the roll motion and the spanwise variation of the streamwise velocity appear to increase as the yaw angle increases from $20^{\circ}$ to $45^{\circ}$ and they then decrease as $\gamma$ increases further from $45^{\circ}$ to $60^{\circ}$. To aid a quantitative comparison of the strength of secondary flow motion in different cases, the total swirling strength $\hat{\lambda}_{c i}$ in the cross-stream plane is presented in Fig. 14. It can be seen that $\hat{\lambda}_{c i}$ peaks at $\gamma=45^{\circ}$.

The average spanwise velocity inside the riblet passages across the span around $x=0.18 m$ can be obtained as follows

$$
\bar{w}=\frac{4 \sin (\gamma)}{\operatorname{sh} \Lambda} \int_{x=0.18}^{x=0.18+s / \sin (\gamma)} \int_{y=-h / 2}^{y=h / 2} \int_{z=0}^{z=0.5 \Lambda} \frac{w}{U_{\infty}} d x d y d z
$$

Using the same method, the average axial velocity of the flow along the riblet passages at the same streamwise location, $\bar{u}_{r}$, can also be calculated. Figure 15 shows the variations of $\bar{w}$ and $\bar{u}_{r}$ with yaw angle $\gamma$. From Fig. 15(a), it can be seen that $\bar{w}$ follows a similar 


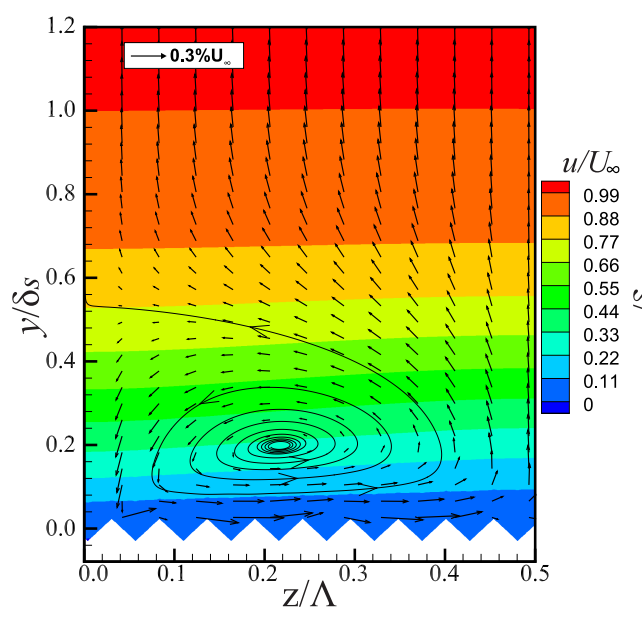

(a) Case 5A: $\gamma=20^{\circ}$

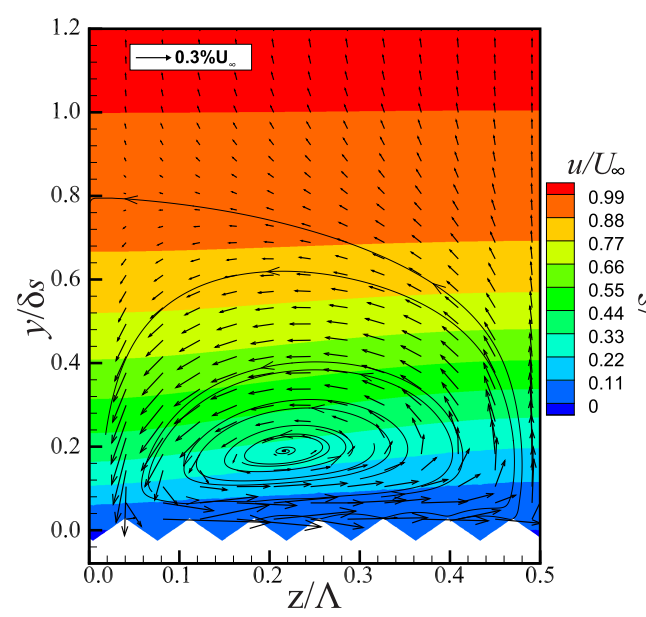

(c) Case 5D: $\gamma=45^{\circ}$

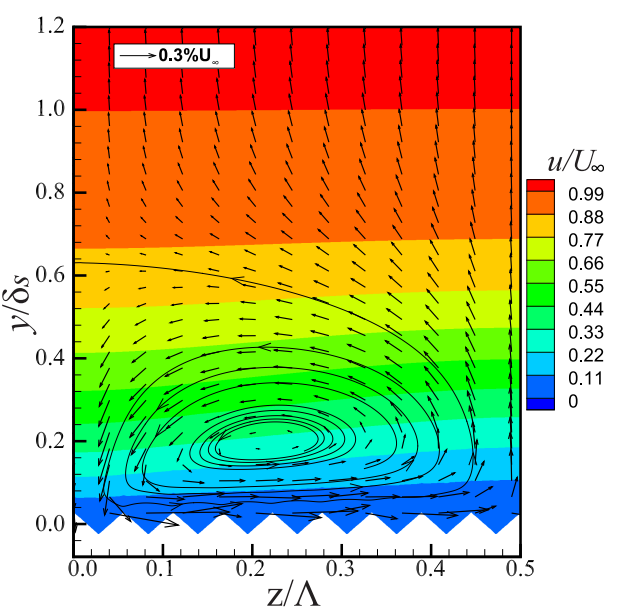

(b) Case $5: \gamma=30^{\circ}$

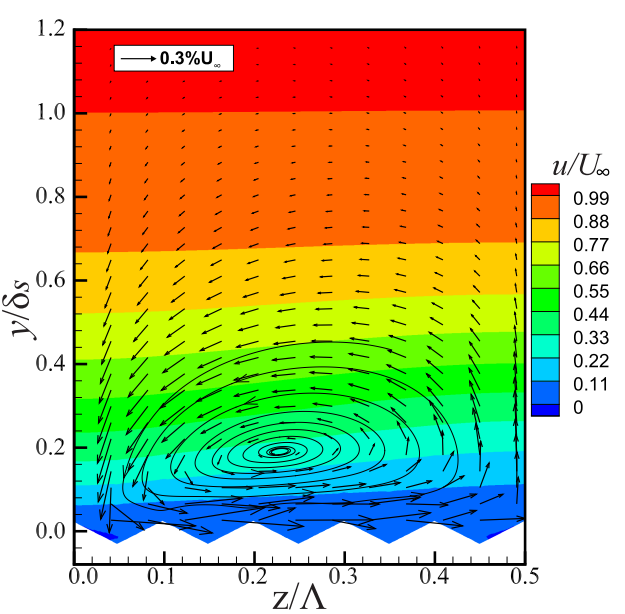

(d) Case 5F: $\gamma=60^{\circ}$

Figure 13: Contours of streamwise velocity and in-plane velocity vector in the cross-stream plane $x=0.18 \mathrm{~m}$ in the cases with different yaw angles. For all cases, $\Lambda / \delta_{s}=1.32, s=0.75 \mathrm{~mm}$ and $h=0.6 \mathrm{~mm}$.

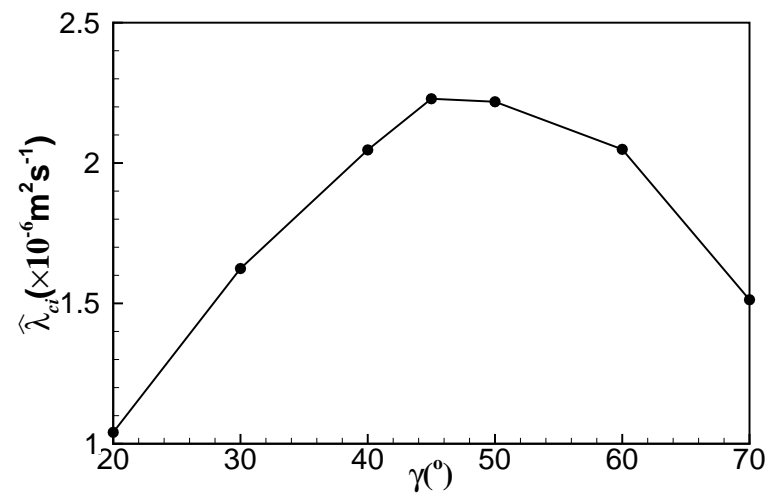

Figure 14: Variations of total swirling strength with yaw angle in the cross section at $x=0.18 \mathrm{~m}$ in the cases with different yaw angles. 
trend as that of total swirling strength shown in Fig. 14. A strong correlation between the strength of secondary flow motion and the magnitude of spanwise velocity is not surprising. In fact, the large-scale secondary flow motion observed in the cross-stream plane is driven by the spanwise flow in the near-wall region induced by the C-D riblets.

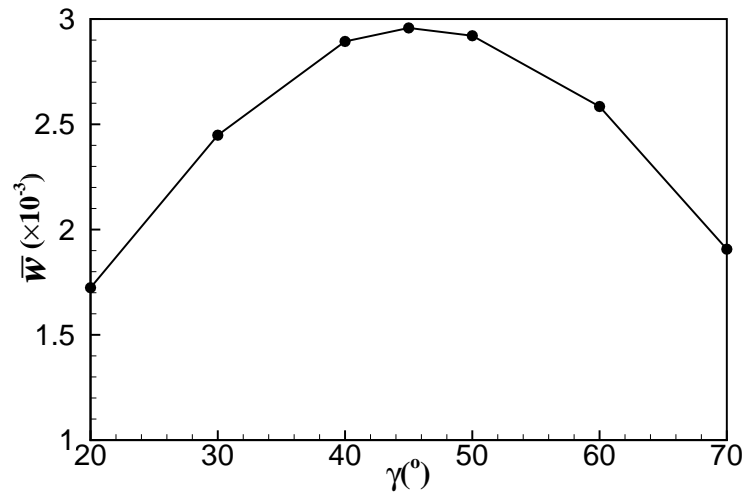

(a) Spanwise velocity

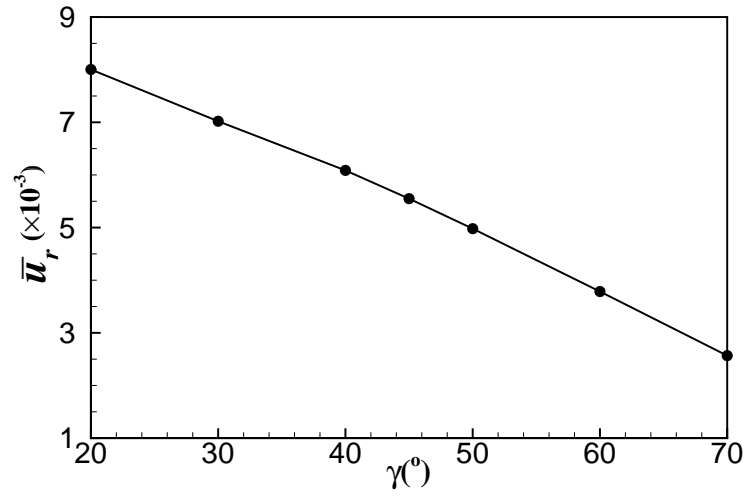

(b) Axial velocity along riblet passage

Figure 15: (a) Average spanwise velocity and (b) Average axial velocity inside the riblet passages around $x=0.18 \mathrm{~m}$ in the cases with different yaw angles. For all cases, $\Lambda / \delta_{s}=1.32, s=0.75 \mathrm{~mm}$ and $h=0.6 \mathrm{~mm}$.

A plausible explanation for the maximum strength of secondary flow motion occurring around $\gamma=45^{\circ}$ is given as follows. Due to the yawed orientation of the riblets relative to the freestream, the surface fluid tends to follow the direction of riblet passages which presents the minimum resistance. If the streamwise velocity of the surface fluid is assumed to be invariant with the yaw angle, the axial velocity of the flow inside the riblet passages, $\bar{u}_{r}$, is expected to vary with yaw angle in a cosine manner exhibiting a monotonic decrease from a maximum value at $\gamma=0^{\circ}$ to zero as $\gamma$ increases to $90^{\circ}$. The linear visual impression of the variation of axial velocity with yaw angle seen in Fig. 15(b) is believed to be caused by truncation of the portion with yaw angle less than $20^{\circ}$ and greater than $70^{\circ}$. The spanwise velocity inside the riblets, $\bar{w}$, which is induced by this axial velocity, can be estimated to be $\bar{u}_{r} * \sin (\gamma)$. Therefore, the spanwise velocity is expected to be correlated with $\cos (\gamma) * \sin (\gamma)$ and reach its maximum at $\gamma=45^{\circ}$, as shown in Fig. 15(a). Furthermore, the strength of secondary flow motion, which depends on the magnitude of the spanwise flow velocity in the near-wall region, is expected to reach its maximum at $\gamma=45^{\circ}$ too. 


\section{Conclusion}

In this paper, a flat-plate laminar boundary layer developing over a convergingdiverging riblet section is studied numerically. After a grid convergence study is completed to confirm the choice of computational mesh, the computational results are validated using experimental data. The nature and development of the secondary flow induced by C-D riblets are then examined. Furthermore, the effects of riblet height, the wavelength and yaw angle on the secondary flow motion generated by the riblets (roll mode) are investigated in detail. Some interesting results are obtained and the finding from this study could be used to inform the choice of riblet geometry for maximizing the control effect in a given boundary layer flow.

It is established from this study that despite their small size C-D riblets induce a roll mode in the cross-stream plane which is capable of generating a substantial modification to the entire boundary layer. The large-scale roll mode is essentially induced by the spanwise velocity produced by yawed riblet passages and hence belongs to the Prandtl's first kind. The strength of the large-scale roll mode strongly depends on the magnitude of the spanwise flow velocity in the near-wall region.

It is found that a larger riblet height induces a stronger secondary flow in the crossstream plane. The exact structure of this secondary flow depends on the relative size of riblet height and wavelength to the local boundary layer thickness. Over the range of parameters examined in this paper, three different patterns are observed. A single vortex located in the mid span is obtained when the wavelength is large and the riblet height is small. Two vortices will coexist in cases with a combination of large riblet height and large wavelength. When the wavelength is small and the riblet height is large, a single vortex closer to the diverging line will be produced.

The strength of the secondary flow in a cross-stream plane increases as the riblet wavelength increases and it levels off at large wavelengths whereas the average strength per unit area exhibits a peak around a ratio between riblet wavelength and local boundary layer thickness of 1.0. With the increase of yaw angle, the strength of the secondary flow increases firstly and then shows a decreasing trend. Near $\gamma=45^{\circ}$, the strength reaches to the peak value. 


\section{Acknowledgements}

The first author would like to acknowledge the sponsorship from The China Scholarship Council and The University of Manchester. He also would like to thank Dr. Fang $\mathrm{Xu}$ for valuable discussions and provision of his experimental data used for validating some of the numerical results presented in this paper.

\section{References}

[1] D. Bechert, M. Bartenwerfer, The viscous flow on surfaces with longitudinal ribs, Journal of fluid mechanics 206 (1989) 105-129.

[2] P. Luchini, F. Manzo, A. Pozzi, Resistance of a grooved surface to parallel flow and cross-flow, Journal of fluid mechanics 228 (1991) 87-109.

[3] M. J. Walsh, Riblets as a viscous drag reduction technique, AIAA journal 21 (4) (1983) 485-486.

[4] D. Bechert, M. Bruse, W. v. Hage, J. T. Van der Hoeven, G. Hoppe, Experiments on drag-reducing surfaces and their optimization with an adjustable geometry, Journal of fluid mechanics 338 (1997) $59-87$.

[5] S.-J. Lee, S.-H. Lee, Flow field analysis of a turbulent boundary layer over a riblet surface, Experiments in fluids 30 (2) (2001) 153-166.

[6] H. Choi, P. Moin, J. Kim, Direct numerical simulation of turbulent flow over riblets, Journal of fluid mechanics 255 (1993) 503-539.

[7] D. Goldstein, R. Handler, L. Sirovich, Direct numerical simulation of turbulent flow over a modeled riblet covered surface, Journal of Fluid Mechanics 302 (1995) 333-376.

[8] O. El-Samni, H. Chun, H. Yoon, Drag reduction of turbulent flow over thin rectangular riblets, International Journal of Engineering Science 45 (2-8) (2007) 436-454.

[9] R. Garcia-Mayoral, J. Jiménez, Hydrodynamic stability and breakdown of the viscous regime over riblets, Journal of Fluid Mechanics 678 (2011) 317-347.

[10] E. v. Bacher, C. Smith, A combined visualization-anemometry study of the turbulent drag reducing mechanisms of triangular micro-groove surface modifications, in: Shear Flow Control Conference, 1985 , p. 548.

[11] K.-S. Choi, Near-wall structure of a turbulent boundary layer with riblets, Journal of fluid mechanics 208 (1989) 417-458.

[12] B. Dean, B. Bhushan, Shark-skin surfaces for fluid-drag reduction in turbulent flow: a review, Philosophical Transactions of the Royal Society A: Mathematical, Physical and Engineering Sciences 368 (1929) (2010) 4775-4806.

[13] W. Hage, D. Bechert, M. Bruse, Yaw angle effects on optimized riblets, in: Aerodynamic Drag Reduction Technologies, Springer, 2001, pp. 278-285.

[14] D. Bechert, M. Bruse, W. Hage, Experiments with three-dimensional riblets as an idealized model of shark skin, Experiments in fluids 28 (5) (2000) 403-412. 
[15] K. Koeltzsch, A. Dinkelacker, R. Grundmann, Flow over convergent and divergent wall riblets, Experiments in fluids 33 (2) (2002) 346-350.

[16] H. Chen, F. Rao, X. Shang, D. Zhang, I. Hagiwara, Flow over bio-inspired 3d herringbone wall riblets, Experiments in fluids 55 (3) (2014) 1698.

[17] B. Nugroho, N. Hutchins, J. P. Monty, Large-scale spanwise periodicity in a turbulent boundary layer induced by highly ordered and directional surface roughness, International Journal of Heat and Fluid Flow 41 (2013) 90-102.

[18] K. Kevin, J. P. Monty, H. Bai, G. Pathikonda, B. Nugroho, J. M. Barros, K. T. Christensen, N. Hutchins, Cross-stream stereoscopic particle image velocimetry of a modified turbulent boundary layer over directional surface pattern, Journal of Fluid Mechanics 813 (2017) 412-435.

[19] B. Nugroho, Highly ordered surface roughness effects on turbulent boundary layers, Ph.D. thesis (2015).

[20] K. Kevin, J. Monty, N. Hutchins, Turbulent structures in a statistically three-dimensional boundary layer, Journal of Fluid Mechanics 859 (2019) 543-565.

[21] F. Xu, S. Zhong, S. Zhang, Vortical structures and development of laminar flow over convergentdivergent riblets, Physics of Fluids 30 (5) (2018) 051901.

[22] F. Xu, S. Zhong, S. Zhang, Statistical analysis of vortical structures in turbulent boundary layer over directional grooved surface pattern with spanwise heterogeneity, Physics of Fluids 31 (8) (2019) 085110. doi:10.1063/1.5110048.

URL https://aip.scitation.org/doi/abs/10.1063/1.5110048

[23] H. Benschop, W.-P. Breugem, Drag reduction by herringbone riblet texture in direct numerical simulations of turbulent channel flow, Journal of Turbulence 18 (8) (2017) 717-759.

[24] Q. Liu, S. Zhong, L. Li, Effects of Bio-inspired Micro-scale Surface Patterns on the Profile Losses in a Linear Cascade, Journal of Turbomachinery (2019) 1-31arXiv:https: //energyresources.asmedigitalcollection.asme.org/turbomachinery/article-pdf/doi/ 10.1115/1.4044612/5427313/turbo-19-1069.pdf, doi:10.1115/1.4044612.

URL https://doi.org/10.1115/1.4044612

[25] P. Quan, S. Zhong, Q. Liu, L. Li, Attenuation of flow separation using herringbone riblets at $\mathrm{M}_{\infty}=5$, AIAA Journal 57 (1) (2018) 142-152. doi:10.2514/1. J057215.

URL https://doi.org/10.2514/1.J057215

[26] P. Erhard, D. Etling, U. Muller, U. Riedel, K. Sreenivasan, J. Warnatz, Prandtl-essentials of fluid mechanics, Vol. 158, Springer Science \& Business Media, 2010.

[27] W. Anderson, J. M. Barros, K. T. Christensen, A. Awasthi, Numerical and experimental study of mechanisms responsible for turbulent secondary flows in boundary layer flows over spanwise heterogeneous roughness, Journal of Fluid Mechanics 768 (2015) 316-347.

[28] J. Zhou, R. J. Adrian, S. Balachandar, T. Kendall, Mechanisms for generating coherent packets of hairpin vortices in channel flow, Journal of fluid mechanics 387 (1999) 353-396.

[29] Y. Wu, K. T. Christensen, Population trends of spanwise vortices in wall turbulence, Journal of Fluid Mechanics 568 (2006) 55-76. 
[30] D. Chung, J. P. Monty, N. Hutchins, Similarity and structure of wall turbulence with lateral wall shear stress variations, Journal of Fluid Mechanics 847 (2018) 591-613. 